\title{
Effect of Saffron Extract and Crocin in Serum Metabolites of Induced Obesity Rats
}

\author{
Fatin Najwa Ramli $\mathbb{D}^{1}$, Azliana Abu Bakar Sajak $\left(\mathbb{D},{ }^{1}\right.$ Faridah Abas $\mathbb{D}^{2,3}$ \\ Zulfitri Azuan Mat Daud $\mathbb{D}^{1},{ }^{1}$ and Azrina Azlan $\mathbb{D D}^{1,4,5}$ \\ ${ }^{1}$ Department of Nutrition and Dietetics, Faculty of Medicine and Health Sciences, Universiti Putra Malaysia, 43400 UPM Serdang, \\ Selangor, Malaysia \\ ${ }^{2}$ Department of Food Science, Faculty of Food Science and Technology, Universiti Putra Malaysia, 43400 UPM Serdang, \\ Selangor, Malaysia \\ ${ }^{3}$ Laboratory of Natural Products, Institute of Bioscience, Universiti Putra Malaysia, 43400 UPM Serdang, Selangor, Malaysia \\ ${ }^{4}$ Research Centre of Excellence for Nutrition and Non-Communicable Disease, Faculty of Medicine and Health Sciences, \\ Universiti Putra Malaysia, 43400 UPM Serdang, Selangor, Malaysia \\ ${ }^{5}$ Laboratory of Halal Science Research, Halal Products Research Institute Universiti Putra Malaysia, 43400 UPM Serdang, \\ Selangor, Malaysia
}

Correspondence should be addressed to Azrina Azlan; azrinaaz@upm.edu.my

Received 11 October 2019; Revised 15 March 2020; Accepted 20 March 2020; Published 9 April 2020

Academic Editor: Lap Ho

Copyright (c) 2020 Fatin Najwa Ramli et al. This is an open access article distributed under the Creative Commons Attribution License, which permits unrestricted use, distribution, and reproduction in any medium, provided the original work is properly cited.

The effect of saffron extract (Crocus sativus L.) and its primary compound crocin was studied on an induced obesity rat model. Our study is aimed at investigating and comparing the metabolite changes in obese and obese treated with saffron extract and crocin and at improving the understanding of the therapeutic effect of saffron extract and crocin. Two different doses of saffron extracts and crocin (40 and $80 \mathrm{mg} / \mathrm{kg}$ ) were incorporated in a high-fat diet (HFD) and were given for eight weeks to the obese rats. The changes in metabolite profiles of the serum were determined using proton nuclear magnetic resonance $\left({ }^{1} \mathrm{H}-\mathrm{NMR}\right)$. Pattern recognition by multivariate data analysis (MVDA) showed that saffron extract and crocin at $80 \mathrm{mg} / \mathrm{kg}$ was the best dosage compared to $40 \mathrm{mg} / \mathrm{kg}$. It also showed that both treatments work in different pathways, especially concerning glucose, lipid, and creatinine metabolism. In conclusion, although the pure compound, crocin, is superior to the saffron crude extract, this finding suggested that the saffron extract can be considered as an alternative aside from crocin in treating obesity.

\section{Introduction}

Obesity is a growing health problem, and indeed, it has become a high-risk factor in many types of noncommunicable diseases such as type 2 diabetes, hypertension, coronary heart disease, stroke, and certain cancers. According to the World Health Organization (WHO), the worldwide prevalence of overweight and obese in the adult population older than 18 years old is $39 \%$ and $13 \%$, respectively [1]. The most common solution introduced by the physician for obese patients is diet and physical activity. However, not all individuals manage to reduce their weight within the healthy body mass index (BMI). Therefore, the introduction of antiobesity drug therapy, such as orlistat (pancreatic lipase inhibitor) or sibutramine (serotonin and noradrenaline inhibitor), is sometimes needed. Despite this, prolonged usage of these drugs to treat obesity may cause harmful side effects in obese patients such as gastrointestinal side effects, reduction in high-density lipoprotein, and elevated blood pressure and pulse rate $[2,3]$.

Currently, there is a surge of interest in implementing the natural product, especially from plants as one of obesity treatment [4]. Saffron, also scientifically known as Crocus sativus $\mathrm{L}$., is a stemless, perennial herb that belongs to the Iridaceae family. Aside from being used in colouring and flavouring agents, saffron also has been widely used in 
traditional medicine as a herbal therapy in the Middle East and Mediterranean countries [5]. There are three main bioactive components in saffron, including volatile agents (safranal), bitter principles (picrocrocin), and dye materials (crocetin and its glycoside, crocin) $[6,7]$.

A study conducted by Mashmoul et al. [8] showed 29\% and $1.9 \%$ of the ethanolic extract of saffron consist of crocin and safranal, respectively. Crocin has been noted to possess pharmacological activities such as antidepression, anticancer, antioxidant, antidiabetic effects, antiatherogenic potential, and antidyslipidemic effect as well as a radical scavenger effect activity [9-15]. Our previous study [8] found that saffron and its bioactive compound crocin have a significant effect in reducing food consumption, the rate of body weight gain, total fat pad and the weight ratio of epididymal fat to the body, plasma triacylglycerol (TAG), and total cholesterol of obese rats. However, the mechanisms of saffron as antiobesity agent remain scarce. Therefore, further preclinical investigation on the potential of saffron for use as a source to develop effective and safe antiobesity drugs seems necessary.

Metabolomics analysis is a bioanalytical technique used to detect and quantify small molecules in a minimal amount of biological samples. Metabolomics analysis is useful to accomplish the comprehensive global view in the complexity of metabolites and their fluctuations in reaction to physiological or pathophysiological stimuli [16]. The primary analytical methods used in metabolomics analysis are ${ }^{1} \mathrm{H}-\mathrm{NMR}$ spectroscopy and mass spectroscopy. ${ }^{1} \mathrm{H}-\mathrm{NMR}$ is widely established in the metabolomics fields as it needs only a little amount of sample, has ease of preparation, and is a nondestructive method and robust. Moreover, the data in the ${ }^{1} \mathrm{H}-$ NMR technique are highly reproducible and quantitative over a wide dynamic range of compounds, in which it can provide useful information about what is happening in the biological system that can give us further understanding of the disease process [17].

Our previous study has revealed the hypolipidemic effect of saffron extract and crocin. However, the mechanism behind the activities and their mechanisms are still limited. Moreover, it would be interesting to study the comparison regarding therapeutics mechanism between crude extract (saffron) with a pure compound (crocin) which has not been reported previously. Based on that limitation, this study is an extension of our previous study. Whereby, by applying ${ }^{1} \mathrm{H}$ NMR metabolomics, this study is aimed (1) at investigating the metabolite changes in obese and obese treated with saffron extract and crocin, (2) at comparing the metabolites changes in obese treated with saffron and crocin, and (3) at providing a better understanding of the therapeutic effect of saffron extract and crocin.

\section{Materials and Methods}

2.1. Chemicals and Standard. Crocin powder and deuterium oxide containing sodium 3-trimethylsilyl-propionate-2,2,3,3,$\mathrm{d} 4$ (TSP) as an internal standard in the ${ }^{1} \mathrm{H}-\mathrm{NMR}$ metabolomics analysis were purchased from Sigma-Chemical Co. (St. Louis, MO, USA). The source of crocin powder was obtained from saffron stigma plant.
2.2. Preparation of Saffron Extract. The saffron (stigma of Crocus sativus L. flower) planted in Khorasan was purchased from Saman Khoshe Tos Co. (Mashhad, Iran). Voucher specimens were deposited at the herbarium, Faculty of Pharmacy, Mashhad University of Medical Sciences. The species were confirmed as Crocus sativus L. with voucher specimen number 134-0319-1. The maceration method was used to extract the stigma of the saffron plant, as described in our previous study [8]. First, $10 \mathrm{~g}$ of dried-ground saffron stigma was soaked in $500 \mathrm{~mL}$ of ethanol $(80 \% \mathrm{v} / \mathrm{v})$ with a magnetic stirrer for three days at room temperature. Then, the mixture was filtered and concentrated under reduced pressure at $40^{\circ} \mathrm{C}$. Next, the ethanol was removed using a rotary evaporator. The resulting extract was later kept at $-80^{\circ} \mathrm{C}$ and lyophilized using a freeze-drying process. Lastly, the lyophilized extract was kept at $-80^{\circ} \mathrm{C}$ until further used. The yield of the extract obtained was 50\% $(w / w)$. Chromatographic identification of bioactive compound in a saffron extract from our previous study found that $1 \mathrm{~g}$ sample of the dried saffron extract contained $290 \mathrm{mg}$ and $19.13 \mathrm{mg}$ of crocin and safranal, respectively [8].

2.3. Preparation of High-Fat Diet. Rats that consumed HFD received $130 \mathrm{~kJ} / 100 \mathrm{~g}$ of food with a composition of $30 \%$ of carbohydrate, $20 \%$ of protein, and $40 \%$ of fat. Rats that consumed normal diet (ND) received $90 \mathrm{~kJ} / 100 \mathrm{~g}$ of food with a composition of $65 \%$ of carbohydrate, $20 \%$ of protein, and $5 \%$ of fat. The composition of the HFD and ND that were given to the animals was summarized in Supplementary Material, Table S1. The primary source of fat used in the HFD was beef tallow. Rats that received ND and HFD were categorized as healthy and disease controls, respectively. The treatment in this study was administered to the rats by combining it with the HFD. Previous studies have reported that both different doses of saffron extract and crocin did not exhibit any side effect and did not cause damage to any major organ $[18,19]$. No lethality was observed in treated rats during eight weeks of treatment period [8]. Therefore, treatment with saffron extract and crocin was provided by two different doses, $40 \mathrm{mg} / \mathrm{kg}$ and $80 \mathrm{mg} / \mathrm{kg}$, as the final dose to be incorporated in the HFD.

2.4. Animal Care and Experimental Design. A total of 42 inbred male Sprague-Dawley rats (body weight $217 \pm 25 \mathrm{~g}$, six weeks old) was purchased from Sapphire Enterprise, Malaysia, and used for the study after obtaining ethics approval from the Institutional Animal Care and Use Committee of the Faculty of Medicine and Health Sciences, Universiti Putra Malaysia (Permit Number: UPM/FPSK/ PADS/BR-UUH/00473). The rats were individually housed in polypropylene cages in an environmentally controlled clean air room under standard laboratory conditions $(12 \mathrm{~h}$ light $/ 12 \mathrm{~h}$ dark cycle, $60 \pm 5 \%$ relative humidity, $22 \pm 2{ }^{\circ} \mathrm{C}$ ). The rats were fed a standard laboratory food pellet diet and allowed water ad libitum for an acclimatization period of 14 days before the experiment. After two weeks of acclimatization, rats were fed a normal diet $(n=6)$ and an HFD $(n=36)$. The weight of obese rats (OB) must have a $50 \%$ increase in the basal weight to be considered obese. 
Following 12 weeks of obesity induction, all rats were randomly divided into six groups $(n=6)$ and received diet as follows: group control (LN): given ND diet; group obese (OB): given HFD diet; group obese and crocin $40 \mathrm{mg} / \mathrm{kg}$ (OBCR40): given $\mathrm{HFD}+$ crocin $40 \mathrm{mg} / \mathrm{kg}$ diet; group obese and crocin $80 \mathrm{mg} / \mathrm{kg}$ (OBCR80): given HFD+crocin $80 \mathrm{mg} / \mathrm{kg}$ diet; group obese and saffron extract $40 \mathrm{mg} / \mathrm{kg}$ (OBSF40): given HFD+saffron extract $40 \mathrm{mg} / \mathrm{kg}$ diet; and group obese and saffron extract $80 \mathrm{mg} / \mathrm{kg}$ (OBSF80): given HFD+saffron extract $80 \mathrm{mg} / \mathrm{kg}$ diet.

The crocin and saffron extracts were added to the HFD at concentrations of 0.14 to $0.30 \%$ and fed to the rats continuously for eight weeks. The dietary crocin and saffron extract level was reset based on changes in body weight. To ensure the rats received $100 \%$ appropriate dosage based on their body weight, the HFD that contained crocin and saffron dosages was first given to the rats' meal, and the remaining HFD without the dosages was added afterwards. The food intake was recorded by measuring the daily food consumption. The body weights of the rats were recorded weekly. After 22 weeks, blood samples were drawn via cardiac puncture for metabolomics analysis. The data of food consumption, body weight, and serum biochemistry had been previously published by Mashmoul et al. [8]. Then, the rats were sacrificed by cardiac puncture under an anaesthetic effect with xylazine and ketamine. A simple schematic diagram of the study is shown in Figure 1.

2.5. Preparation of Serum Samples for ${ }^{1} H$-NMR Analysis. The serum samples were prepared using a method as described by Beckonert et al. [20] with slight modifications. Since the present study only focused on the saffron extract and its bioactive compound, crocin, the groups of obese rats that were analyzed by metabolomics analysis were LN, OB, OBSF40, OBSF80, OBCR40, and OBCR80. Only $n=5$ rats from each group was randomly chosen for modelling due to outliers (sick, death) and to standardize the number of rats in each group. Firstly, serum samples were thawed at room temperature. Then, the samples were centrifuged at $3000 \mathrm{~g}$ for $10 \mathrm{~min}$ at $4^{\circ} \mathrm{C}$. The supernatant $(300 \mu \mathrm{L})$ was mixed with $600 \mu \mathrm{L}$ of $0.9 \%$ saline solution (deuterium oxide: water $\left.\left(\mathrm{D}_{2} \mathrm{O}: \mathrm{H}_{2} \mathrm{O}\right)\right)$ containing $0.1 \%$ sodium 3 -trimethylsilyl-propionate-2,2,3,3,-d4 (TSP) as an internal standard at a chemical shift of $\delta 0.0 \mathrm{ppm}$. Next, the final volume was transferred into a $5.0 \mathrm{~mm}$ NMR tube (Norell, Landisville, NJ, USA). Samples were analyzed with the first increment of NOESY pulse sequence and CPMG (Carr-Purcell-Meiboom-Gill) pulse sequence with water suppression.

2.6. NMR Measurement and Spectra Processing. NMR spectra of serum samples were performed using $500 \mathrm{MHz}$ Varian INOVA NMR (Varian Inc., California, USA) spectroscopy functioning at frequency $499.91 \mathrm{MHz}$, equipped with a $5.0 \mathrm{~mm}$ TXI probe and maintained at $25^{\circ} \mathrm{C}$. A standard one-dimensional pulse sequence was performed using the first increment of the NOESY pulse sequence (recycle delay $-90^{\circ}-t_{1}-90^{\circ}-t_{\mathrm{m}}-90^{\circ}$-acquisition) to achieve water presaturation. Additionally, a Carr-Purcell-Meiboom-Gill (CPMG) pulse sequence was obtained to enhance the contri- bution of low molecular weight metabolites by suppressing large signals from macromolecules. The mixing time $\left(t_{\mathrm{m}}\right)$ used for a standard one-dimensional experiment was $100 \mathrm{~ms}$. The CPMG spectra were acquired with 128 transients, and common recycle delay of $2.0 \mathrm{~s}$ was used for each sample. Water signal irradiation was applied during the recycle delay. The NMR spectra were manually phased and baseline-corrected calibrated to TSP at $\delta 0.00 \mathrm{ppm}$ using Chenomx software (version 6.2, Alberta, Canada). Next, each of the processed NMR spectra $(\delta 0-10 \mathrm{ppm})$ was segmented into 250 regions with an equal $0.04 \mathrm{ppm}$ via the profiler module. The residual water regions of $\delta 4.53-\delta 5.15 \mathrm{ppm}$ were excluded before analysis to remove any spurious effects of water suppression. Then, the ${ }^{1} \mathrm{H}-\mathrm{NMR}$ output data were exported from Microsoft Excel and imported to SIMCA (version 14.0, Umetrics, Umea, Sweden) for multivariate data analysis (Pareto-scaled).

2.7. Statistical Analysis. First, the data were examined using nonsupervised multivariate data analysis which was principal component analysis (PCA) to explore the intrinsic variation within a group and to assess clustering patterns between groups. Next, the partial least square discriminant analysis (PLS-DA) model of NMR spectral data was also performed to maximize the variations and to determine potential metabolites that contributed to the variation. The validity of the models was assessed by determining the $R^{2}$ and $Q^{2}$ (goodness of prediction parameter) values, while the validity of the models was tested using a permutation test (100 cycles) and misclassification probability test. The metabolite in the serum samples was expressed as mean \pm standard error of the mean. IBM SPSS version 21.0 (Chicago, USA) software was also used to perform one-way ANOVA with Tukey comparison post hoc test to determine the significant difference of the mean value of metabolite concentrations $(p<0.05)$ in each sample for the six groups subjected to the ${ }^{1} \mathrm{H}-\mathrm{NMR}$ metabolomics analysis.

\section{Results}

3.1. Body Weight, Food Consumption, and Serum Biochemistry. The food consumption and body weight of LN, OB, OBCR40, OBCR80, OBSF40, and OBSF80 rat groups which had been previously published [8] were shown in supplementary materials. From Table S2, it is showed that OBCR80 and OBSF80 groups significantly reduced food consumption during eight weeks of the treatment period. Meanwhile, in Figure S1, there are no apparent changes in the body weight between groups. However, there was a trend in the body weight loss for the treatment group, especially for OBCR80 and OBSF80. The effect of saffron extract and crocin on biochemical parameters was also summarized in Supplementary Table S3 as in our previous work $[8,21]$. The OBCR80 group significantly had a lower level of fasting blood glucose, triglycerides (TG), and total cholesterol (TC). As opposed to the OB group, OBSF80 also significantly had a lower concentration of fasting blood glucose and TC. 


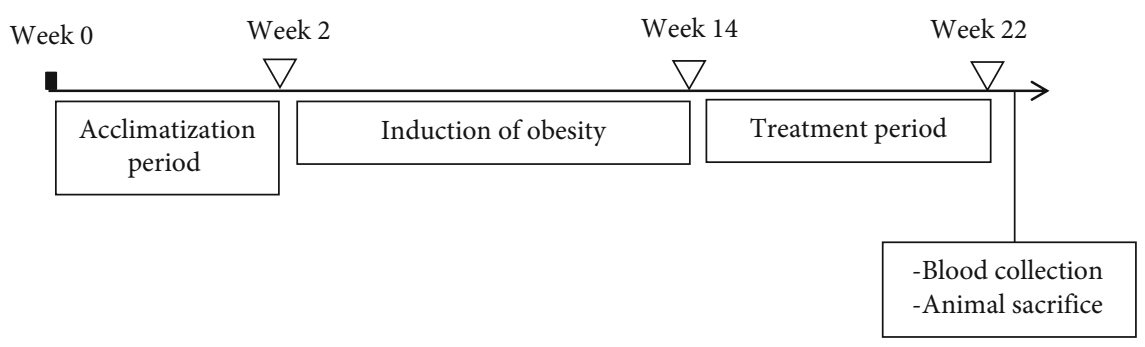

Figure 1: Animal study timeline.

3.2. ${ }^{1} H$-NMR Serum Spectra of the Nontreated and Treated Groups. Representative ${ }^{1} \mathrm{H}-\mathrm{NMR}$ CPMG spectra of serum from LN, OB, OBCR80, and OBSF80 rat groups were shown in Supplementary Material, Figure S2, with primary metabolites assigned. For treatments, only OBCR80 and OBSF80 were picked as a representative, since they exerted a better activity compared to $40 \mathrm{mg} / \mathrm{kg}$ (explained further in the next section). The metabolites were assigned based on a database provided in Chenomx NMR Suite Software 9 (version 6.1, Alberta, Canada). The metabolites should have the same shape splitting or coupling constant, and within the chemical shift range, further identification and confirmation also had been made via chemical shift and coupling constant from Human Metabolome Database (HMDB) and published assignments. A list of the identified metabolites, including their chemical shifts, is represented in Supplementary Material, Table S4.

3.3. Multivariate Data Analysis of Processed NMR Spectra. The treatment on obese rats was started by incorporating the saffron extract or crocin in the HFD on the $13^{\text {th }}$ week for eight weeks. First, a general PCA model consists of all groups (LN, OB, OBSF40, OBSF80, OBCR40, and OBCR80) was performed for a general visualization of group clustering and outlier detection. Even though no outlier was found in the model, no apparent clustering or grouping can be noted in the model (even in another supervised model, PLS-DA). This is due to the increase of variability as there are too many groups in one model. Therefore, it was decided to separate the type of treatment (saffron extract and crocin) in two different models, and the best dosage from each treatment was selected from each model. The best treatment was decided based on (1) a clear separation from the OB group and (2) supported by the data in biochemistry test.

The first treatment model by the saffron extract was built by using PLS-DA model (Figure 2, $R^{2} Y_{\text {cum }}=0.642, Q_{\text {cum }}^{2}$ $=0.272$ ). A permutation test with 100 permutation cycles (Supplementary Material: Figures S3, S4, S5, and S6) and misclassification probability test (Supplementary Material: Table S5) were performed to validate the PLS-DA model. The model exhibited clear clustering of the LN, OB, OBSF40, and OBSF80 groups. From the model, two out of five rats treated from the OBSF80 group shifted to the same principal component 1 (PC1) with the LN group. However, the changes did not manage to revert the OBSF80 into the same quadrant with LN (lower right side of PC1). The changes of OBSF 80 can be notably being seen in metabolites such as lactate, pyruvate, glucose, taurine, alanine, betaine, TMAO, and creatinine from loading score scatter plot.

In the second treatment model involving crocin, the PLSDA model (Figure 3) also was being performed in ${ }^{1} \mathrm{H}-\mathrm{NMR}$ dataset of LN, OB, OBCR40, and OBCR80, which resulted in a PLS-DA model with the goodness of fit $R^{2} Y_{\text {cum }}=0.747$ and $Q^{2}$ cum $=0.355$. A permutation test with 100 permutation cycles (Supplementary Material: Figures S7, S8, S9, and S10) and misclassification probability test (Supplementary Material: Table S6) were also conducted to validate the PLS-DA model. From the result, three out of five rats treated from the OBCR80 group shifted to the same PC1 with the LN group. The OBCR80 group manages to move close to the same quadrant with LN (lower right side of PC1). The changes of OBCR80 can be notably seen in metabolites such as pyruvate, glucose, taurine, 3-HB, betaine, and TMAO from loading score scatter plot. These results are also being supported by our previous biochemical results by Mashmoul et al. $[8,21]$ where the OBCR80 group significantly had a lower content of fasting blood glucose, TG, TC, insulin, and leptin with a higher content of adiponectin, ghrelin, and catalase as opposed to the $\mathrm{OB}$ group. Therefore, the best amount of crocin that manages to revert specific biochemical parameters and metabolites close to the LN group is at $80 \mathrm{mg} / \mathrm{kg}$ as compared to OBCR40.

A combination of the best extract from each treatment (OBCR80 and OBSF80) was compared in the new PLS-DA model (Figure 4). A model with the goodness of fit $R^{2} Y_{\text {cum }}$ $=0.477$ and $Q_{\text {cum }}^{2}=0.110$ with validity by 100 permutation cycles (Supplementary Material: Figures S11, S12, S13, and S14) and misclassification table (Supplementary Material: Table S7) with $p<0.05$ that showed the goodness of fit of the model is moderate. From the model, the OBCR80 is grouped near to LN. This result indicates that half of the rats treated with crocin were able to revert to LN. The changes are notable, especially in pyruvate, glucose, taurine, betaine, and TMAO metabolites. The differences of metabolite level, especially in 3-HB in OBCR80 and OBSF80, discriminate OBSF80 from LN, even though most metabolite changes in OBSF80 is comparable to LN. All of these results suggest that crude extract saffron and crocin works in different pathways.

3.4. Pathway Analysis. For a better understanding, a summarized Table 1 and illustrated schematic diagram and pathways involving the metabolites affected were presented 


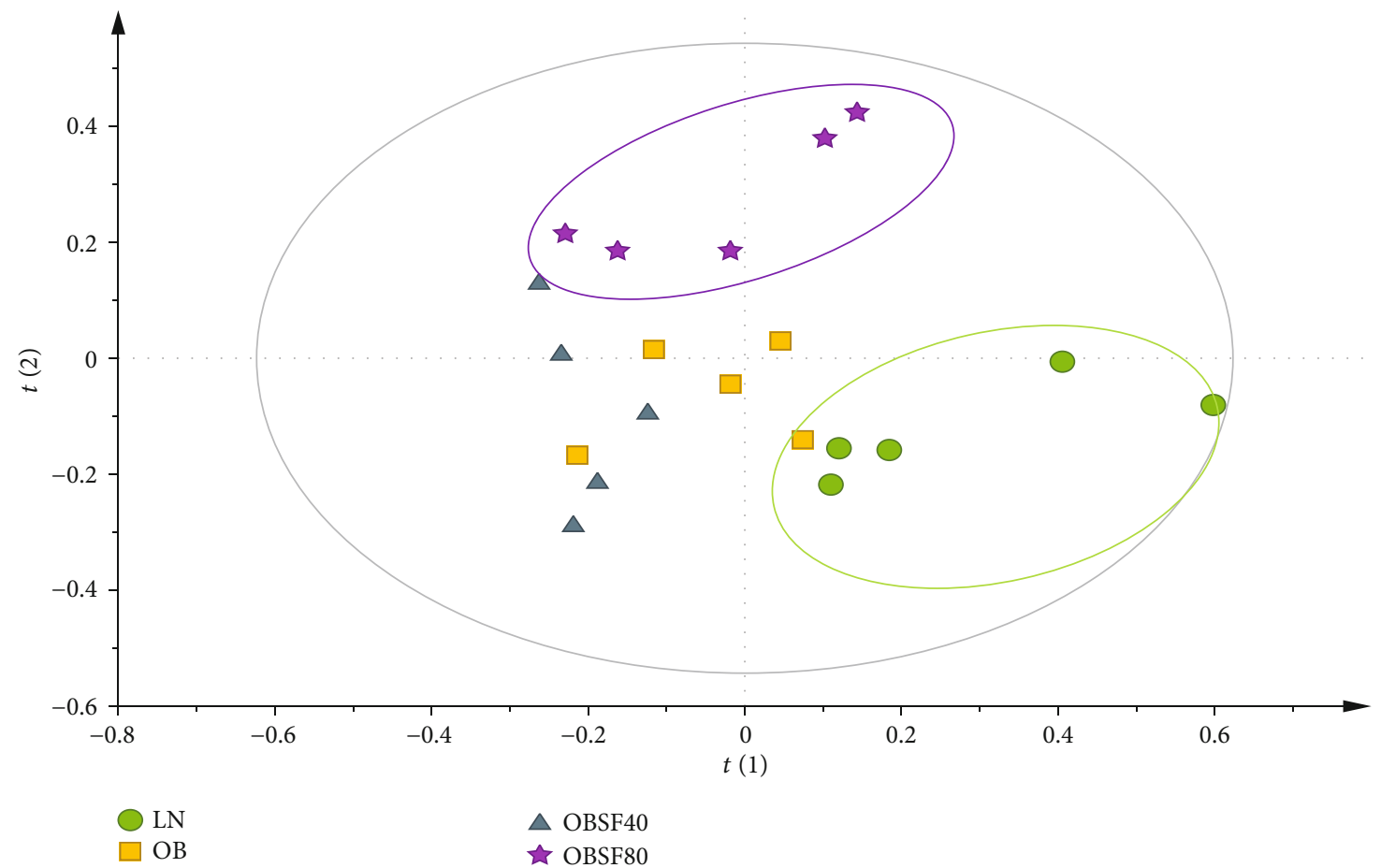

(a)

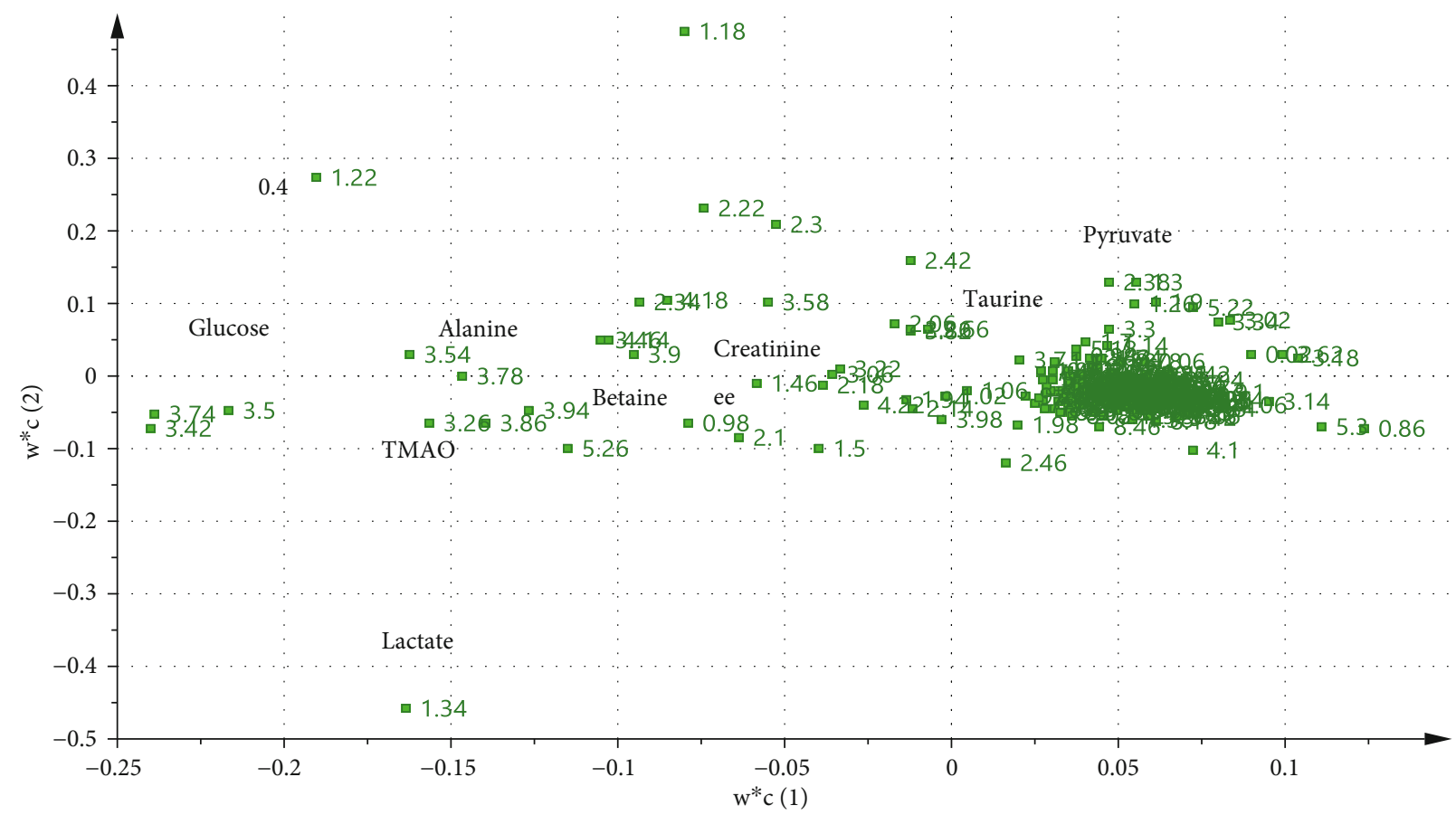

(b)

FIGURE 2: PLS-DA-derived (a) score plot and (b) loading score plot obtained using ${ }^{1} \mathrm{H}$-NMR spectra of serum samples in Sprague-Dawley rats from the lean (LN), obese (OB), obese+saffron extract $40 \mathrm{mg} / \mathrm{kg}$ (OBSF40), and obese saffron extract $80 \mathrm{mg} / \mathrm{kg}$ (OBSF80) groups after eight weeks of the treatment period $\left(R^{2} Y_{\text {cum }}=0.642, Q_{\text {cum }}^{2}=0.272\right)$.

(Figures 5-7). A cutoff value of more than 0.7 for the variable importance in project (VIP) is generally acceptable to identify metabolites contributing to the clustering of the different groups. The pathway was built based on literature [22], Kyoto Encyclopedia of Genes and Genomes (KEGG) path- way [23], and MetaboAnalyst 4.0 [24, 25]. Tables 2 and 3 are the summarized list of pathways found by Qualitative MetPA analysis using MetaboAnalyst 4.0 in OBSF80 and OBCR80. A total of 17 pathways were involved in OBSF80 as compared to OBCR80, in which only 15 pathways were 


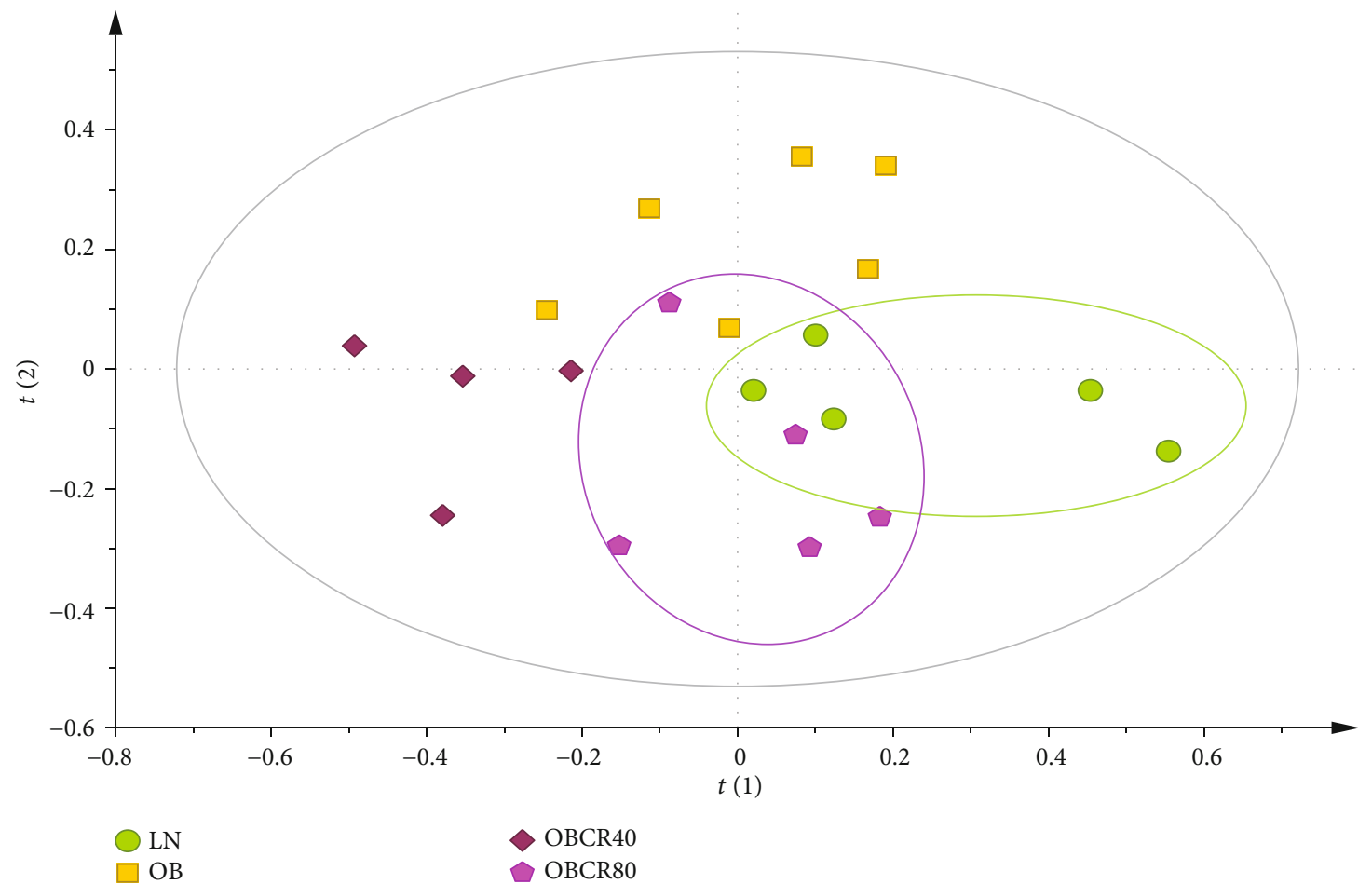

(a)

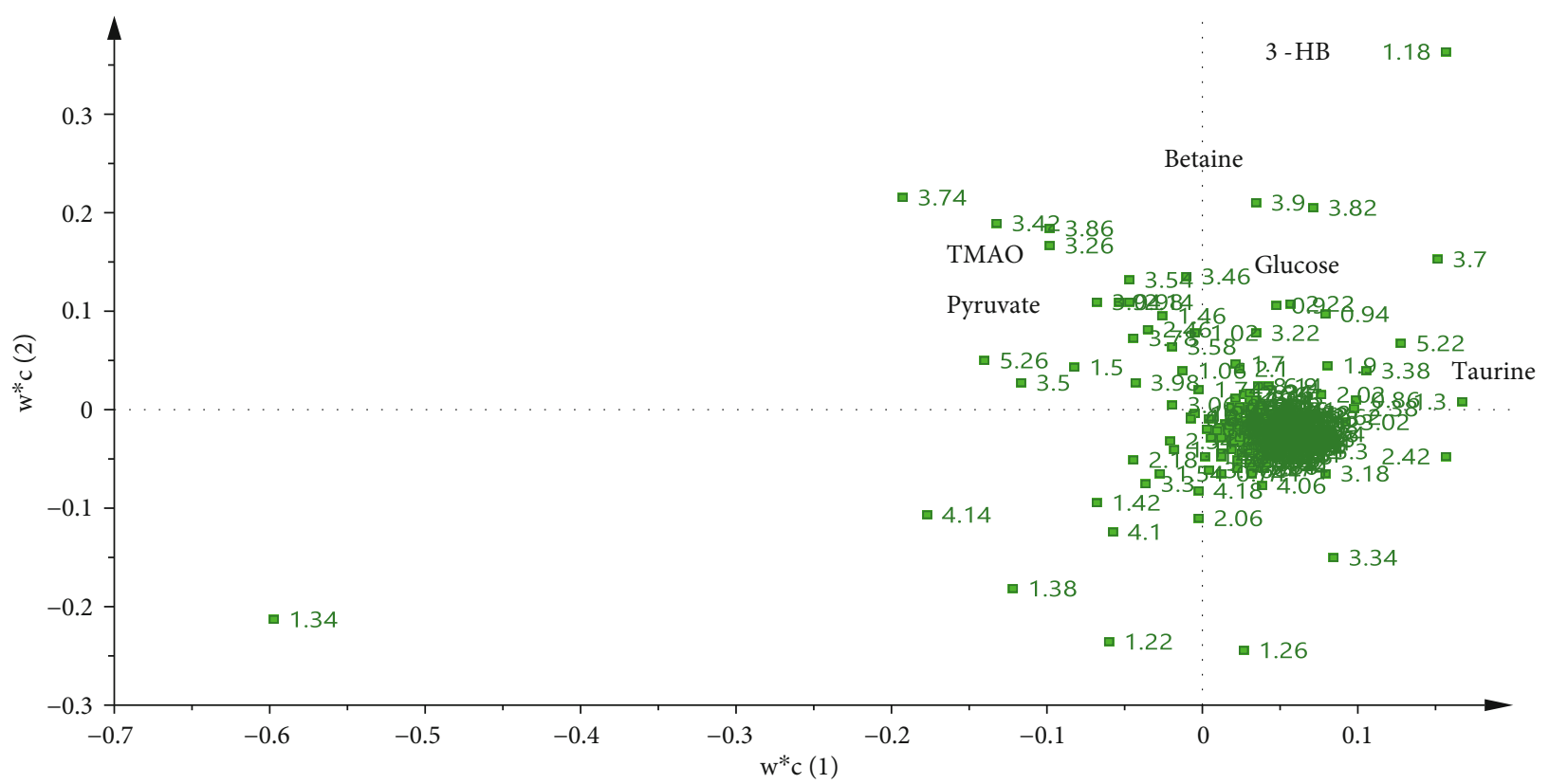

(b)

FIGURE 3: PLS-DA-derived (a) score plot and (b) loading score plot obtained using ${ }^{1} \mathrm{H}$-NMR spectra of serum samples in Sprague-Dawley rats from lean (LN), obese (OB), obese+crocin $40 \mathrm{mg} / \mathrm{kg}$ (OBCR40), and obese+crocin $80 \mathrm{mg} / \mathrm{kg}$ (OBCR80) groups after eight weeks of the treatment period $\left(R^{2} Y_{\text {cum }}=0.747, Q_{\text {cum }}^{2}=0.355\right)$.

involved. The outcome showed that saffron and crocin were mostly involved in glucose and energy metabolism, lipid metabolism, amino acid metabolism, and gut microbiota. Note that the changes in metabolite level of OBSF80 are comparable to the LN group compared to OBCR80. The only notable difference in OBSF80 compared to the LN group is the 3-hydroxybutyrate level.

\section{Discussion}

Studies related to the effects of saffron extract and its bioactive compound crocin in metabolic syndrome-related diseases are not relatively new. The previous study which involves saffron extract and crocin reveals that they managed to improve appetite, dietary consumptions, and body composition in patients 


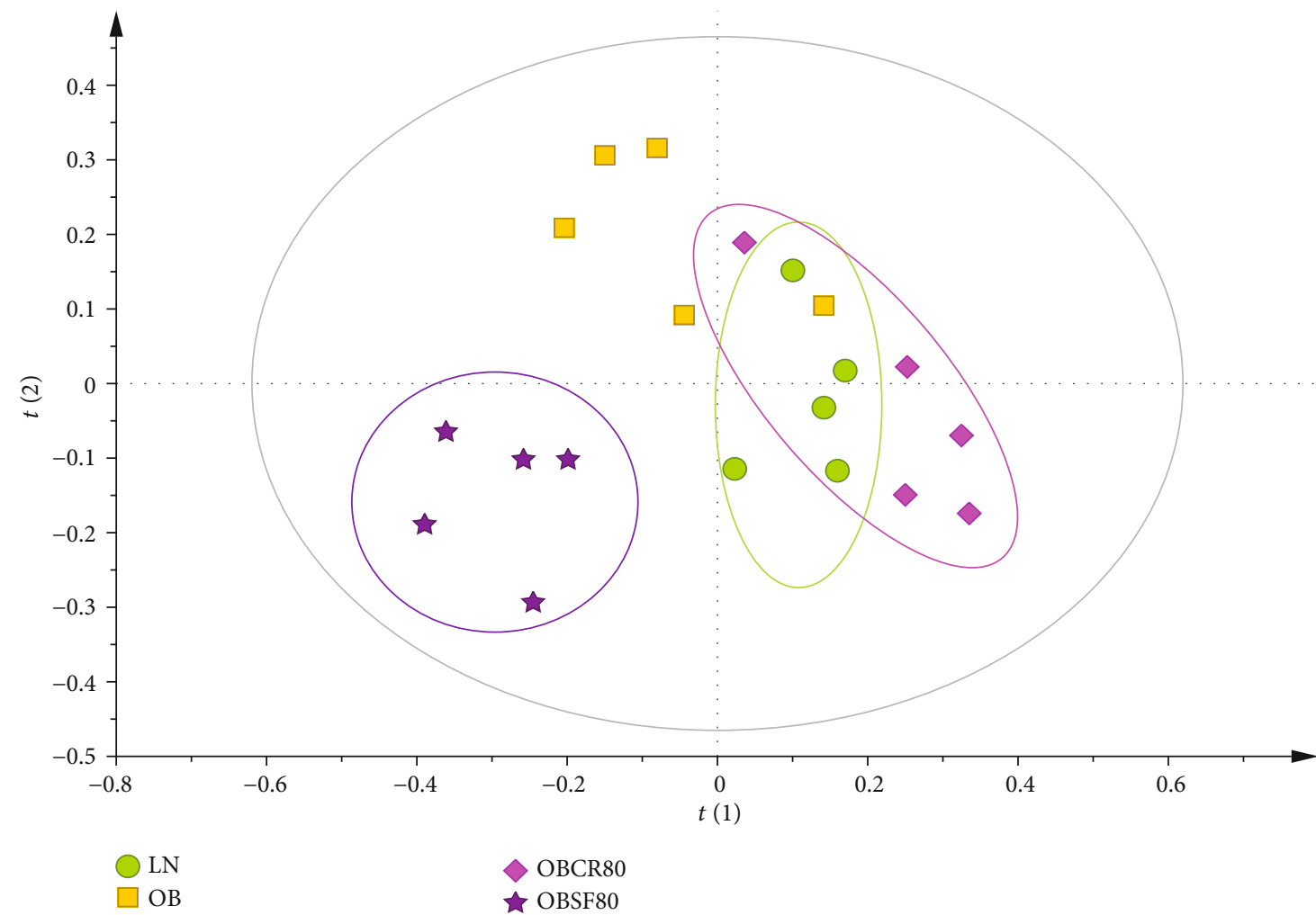

(a)

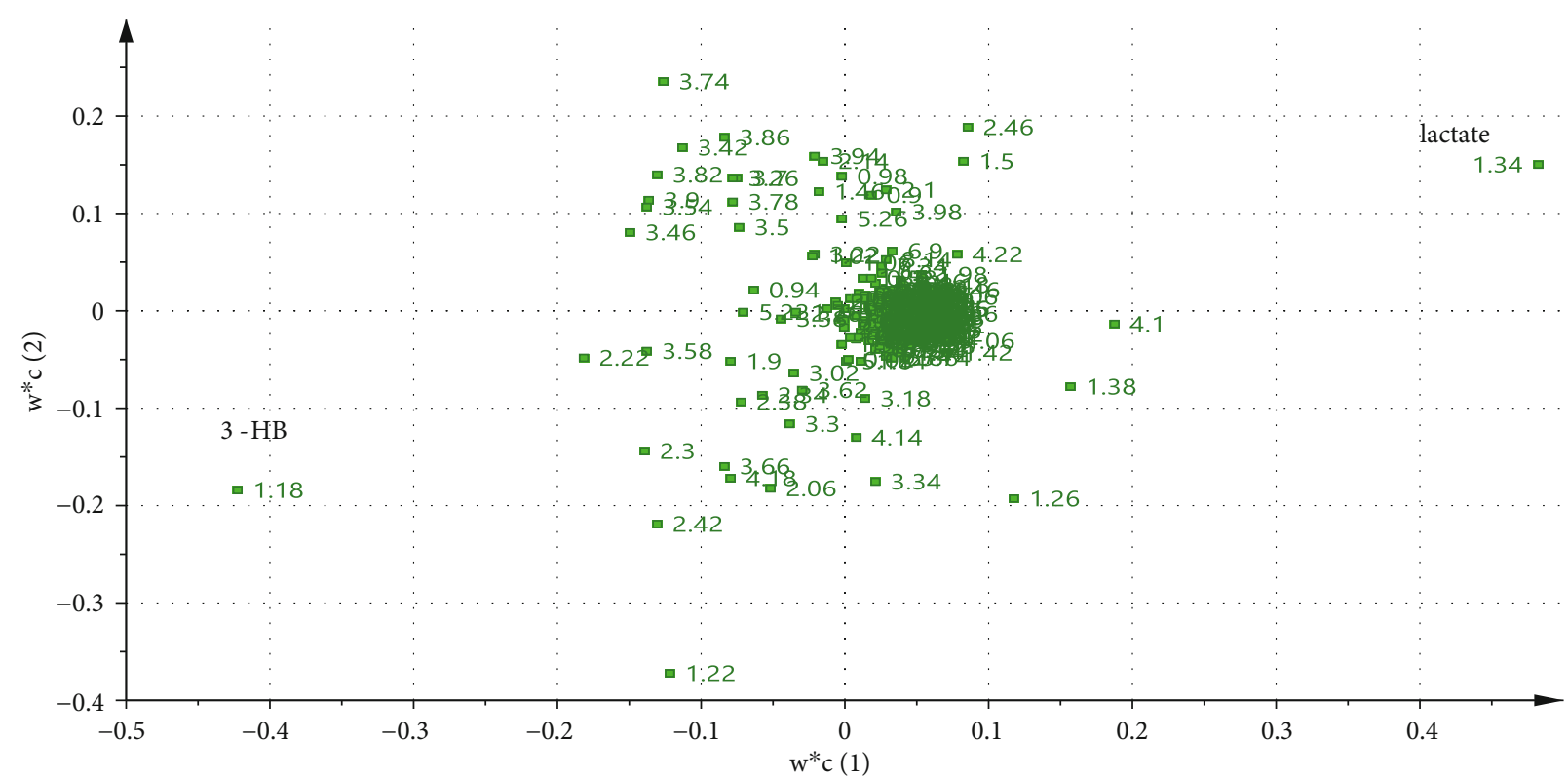

(b)

FiguRE 4: PLS-DA-derived (a) score plot and (b) loading score plot obtained using ${ }^{1} \mathrm{H}-\mathrm{NMR}$ spectra of serum samples in Sprague-Dawley rats from the lean (LN), obese (OB), obese+saffron extract $80 \mathrm{mg} / \mathrm{kg}$ (OBSF80), and obese+crocin $80 \mathrm{mg} / \mathrm{kg}$ (OBCR80) groups after eight weeks of the treatment period $\left(R^{2} Y_{\text {cum }}=0.477, Q_{\text {cum }}^{2}=0.110\right)$.

with coronary artery disease [26]. Interestingly, there also a few reports stating that it can also increase satiety, which eventually reduces food intake [8]. However, the mechanism of saffron and its bioactive compound crocin is still limited [8]. Therefore, new insights are available into the related mechanisms involved, comparing the proper effect of pure compound and crude extract.

4.1. Bodyweight, Food Consumption, and Serum Biochemistry. In this study, it was estimated that the crocin concentration 
TABLE 1: Relative quantification of significance discriminating metabolites based on the concentration of $0.1 \%$ of 3-trimethysilyl propionic2,2,3,3-d4 acid sodium salt (TSP) as an internal standard and quantified using the Chenomx NMR Suite (metabolites were chosen based on combination model, Figure 4).

\begin{tabular}{|c|c|c|c|c|c|c|c|}
\hline Metabolites (mM/L) & Chemical shifts & VIP 1 & VIP 2 & $\mathrm{OB}$ & LN & OBCR80 & OBSF80 \\
\hline Lactate & 1.34 & 7.41 & 5.70 & $153.71 \pm 32.5$ & $67.2 \pm 37.0^{* *}$ & $110.2 \pm 68.2^{+}$ & $69.2 \pm 16.6^{* *}$ \\
\hline Pyruvate & 2.38 & 1.11 & 1.20 & $5.4 \pm 2.2$ & $10.6 \pm 4.5^{*}$ & $17.2 \pm 8.8^{* *}$ & $21.2 \pm 6.5^{* *}$ \\
\hline Glucose & $3.25-5.22$ & 2.31 & 2.91 & $109.5 \pm 27.7$ & $58.0 \pm 31.4^{* *}$ & $41.0 \pm 5.9^{* *}$ & $39.9 \pm 8.7^{* *}$ \\
\hline Taurine & $2.42,3.38$ & 2.00 & 2.54 & $19.9 \pm 11.7$ & $51.1 \pm 15.3^{*}$ & $53.5 \pm 15.3^{* *}$ & $38.3 \pm 13.4^{*}$ \\
\hline 3-Hydroxybutyrate & $1.18,4.14,2.31,2.42$ & 6.50 & 5.11 & $18.6 \pm 4.2$ & $29.8 \pm 9.1^{*}$ & $41.4 \pm 14.6^{* *}$ & $50.5 \pm 28.8^{*+}$ \\
\hline Alanine & $3.78,1.48$ & 1.26 & 1.73 & $24.4 \pm 5.7$ & $14.6 \pm 6.8^{*}$ & $26.5 \pm 2.4^{+}$ & $9.7 \pm 5.3^{* *}$ \\
\hline Betaine & 3.3 & 1.19 & 1.20 & $18.1 \pm 10.6$ & $6.9 \pm 5.6^{*}$ & $10.7 \pm 4.8^{* *}$ & $8.3 \pm 6.9^{*}$ \\
\hline TMAO & 3.26 & 1.20 & 1.71 & $30.4 \pm 15.6$ & $11.1 \pm 4.6^{*}$ & $10.8 \pm 3.9^{*}$ & $10.5 \pm 4.1^{*}$ \\
\hline Creatinine & $3.06,4.06$ & 0.81 & 1.62 & $11.4 \pm 3.5$ & $5.9 \pm 2.3^{* *}$ & $10.4 \pm 3.5^{+}$ & $5.7 \pm 1.9^{* *}$ \\
\hline
\end{tabular}

Values are expressed as the mean \pm SE (standard error of the mean) of five rats; ${ }^{*} p<0.05$ vs. negative control $(\mathrm{OB}),{ }^{* *} p<0.01$ vs. negative control $(\mathrm{OB})$, ${ }^{+} p<0.05$ vs. normal control (LN), and ${ }^{++} p<0.01$ vs. normal control (LN).

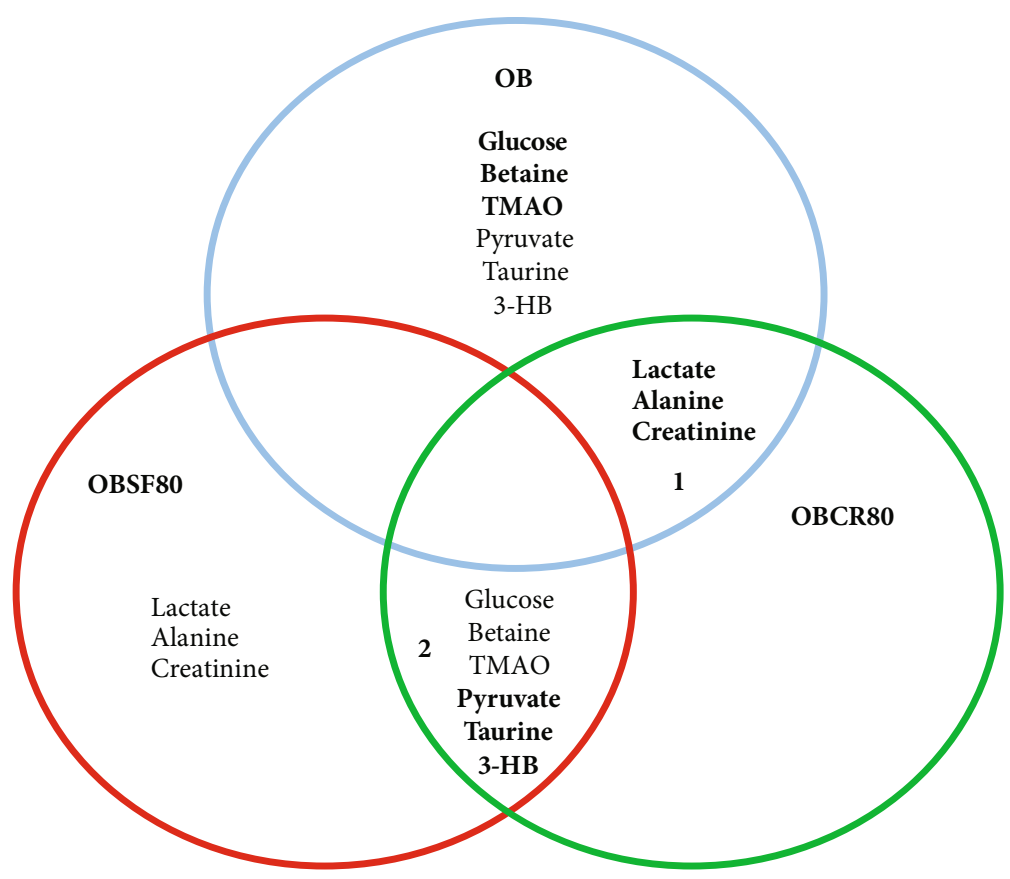

FIGURE 5: Summary of metabolite changes in obese (OB), obese+saffron extract $80 \mathrm{mg} / \mathrm{kg}$ (OBSF80), and obese+crocin $80 \mathrm{mg} / \mathrm{kg}(\mathrm{OBCR} 80)$, where metabolite levels indicated in bold are increased, and those not in bold are decreased in the respective group. Numbers in the overlapped region represent the metabolites shared by the group; 1: OB and OBCR80; 2: OBCR80 and OBSF80.

received in the animal diets of high-dose $(80 \mathrm{mg} / \mathrm{kg})$ and lowdose $(40 \mathrm{mg} / \mathrm{kg})$ saffron extract-treated groups was 23.3 and $11.6 \mathrm{mg}$ of crocin per kilogram of body weight per day. The amounts of crocin received by these groups (saffron-treated groups) are lower than the pure compound-treated group, crocin. When comparing between groups treated with crocin and saffron extract, the results showed that there were no significant differences between the treatment, except in OBSF80 and OBCR80. Theoretically, if the crocin alone is the bioactive compounds in the OBSF80, the result in OBSF80 should be halves (or worse) than the OBCR40 result, as OBCR40 is onefold higher than OBSF80 in terms of crocin concentration. However, based on the biochemistry result (Table S3), the serum biochemistry result from OBSF80 did not differ much compared to OBCR40, except in TC and highdensity lipoprotein (HDL) level. Therefore, we postulate that in the OBSF80 group, all the bioactive compounds in saffron extract (crocin, safranal, picrocrocin, etc.) might synergistically be involved in the saffron-treated group.

In terms of toxicity, both different doses of saffron extract and crocin did not exhibit any side effect and did not cause damage to any major organ $[18,19]$. No lethality was observed 


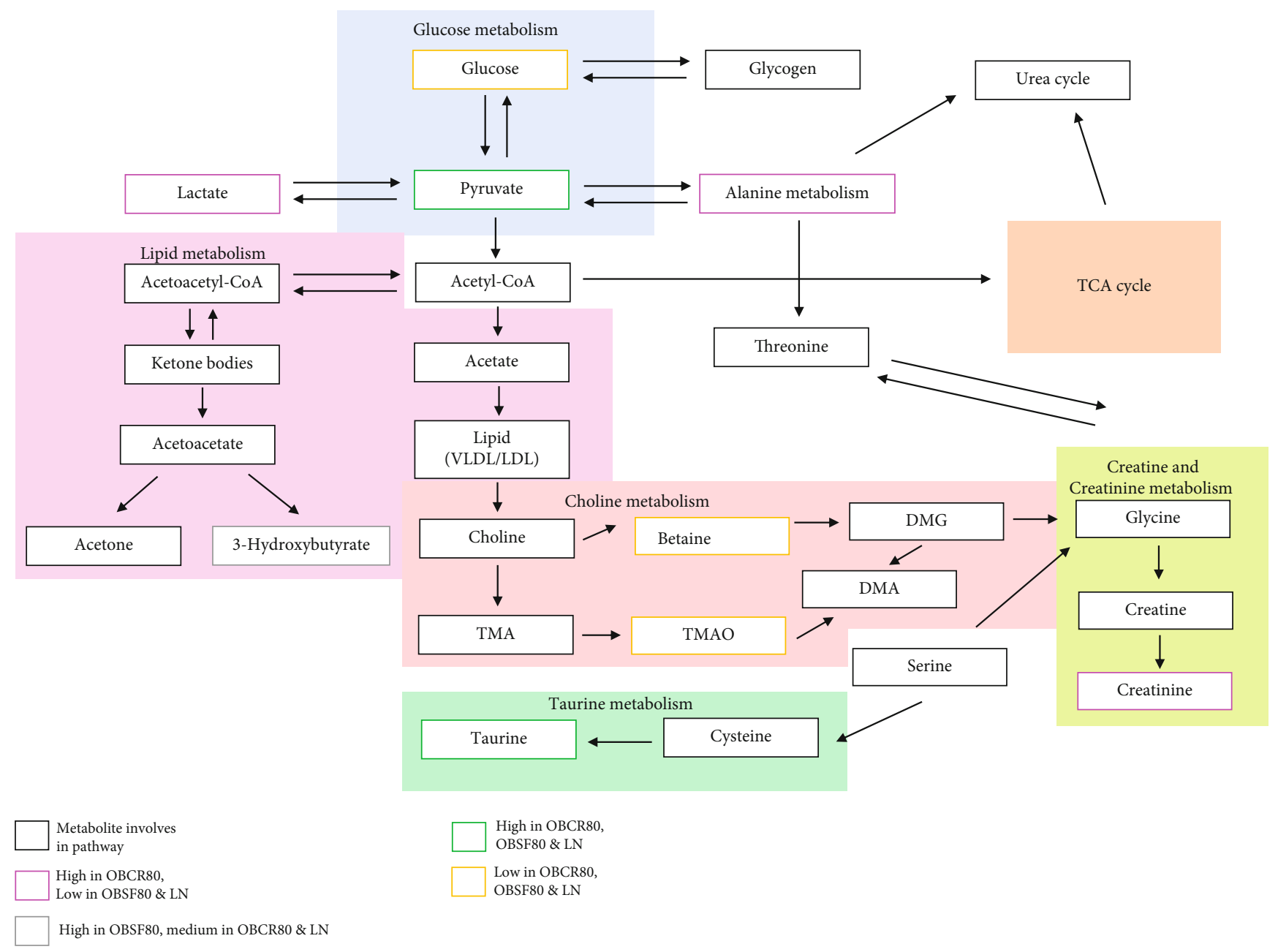

FiguRE 6: Tentative disturbed metabolism and pathways in lean (LN), obese+saffron extract $80 \mathrm{mg} / \mathrm{kg}$ (OBSF80), and obese+crocin $80 \mathrm{mg} / \mathrm{kg}$ (OBCR80).
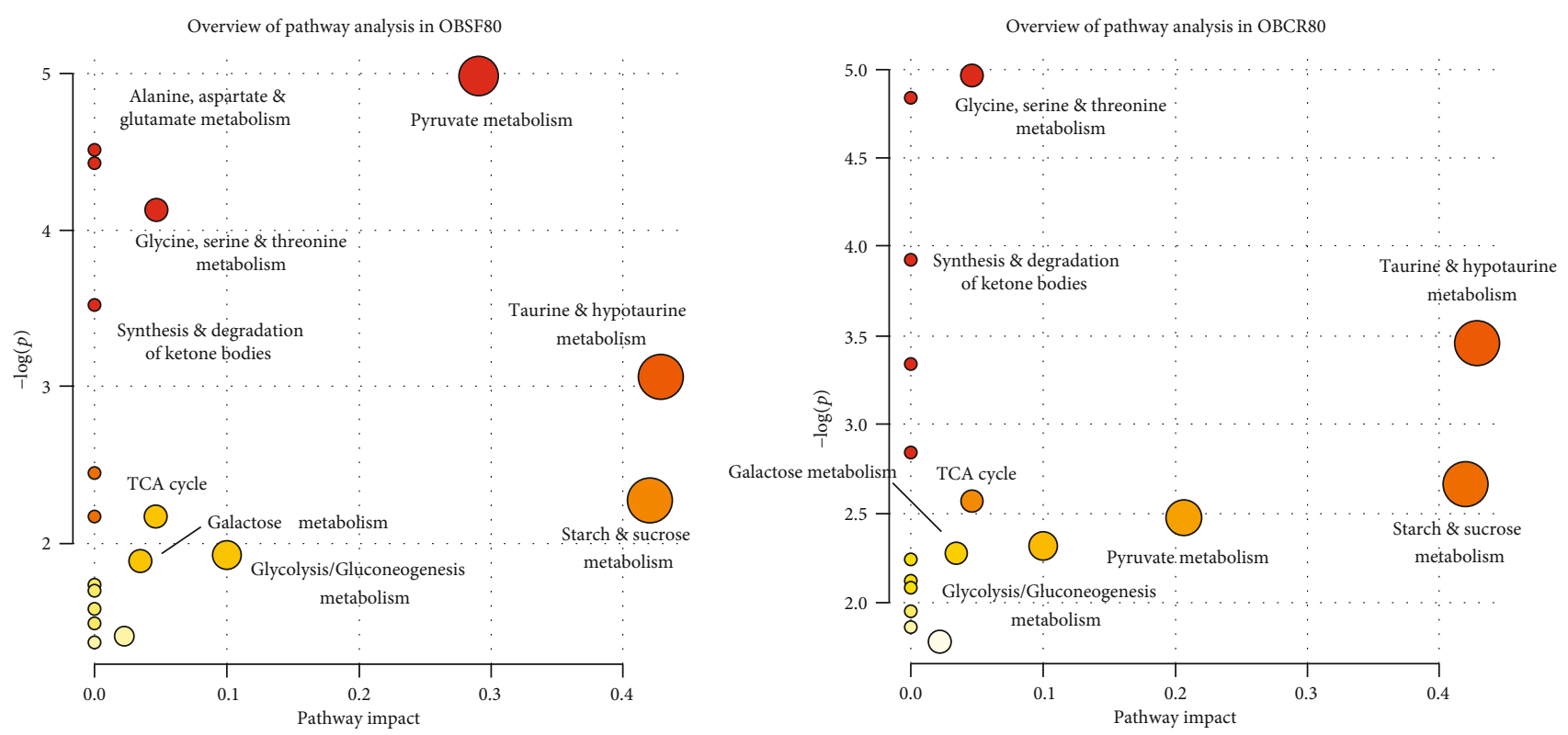

FIGURE 7: Simplified schematic of some pathways as identified by MetaboAnalyst 4.0 in the (a) OBSF80 group and the (b) OBCR80 group. 
TABLE 2: List of pathways found by qualitative MetPA analysis using MetaboAnalyst 4.0 in OBSF80.

\begin{tabular}{|c|c|c|c|c|c|c|c|}
\hline Ranking & Pathway & Total & Expected & Hits & $\%$ found & Raw $p$ & Impact \\
\hline 3 & Pyruvate metabolism & 22 & 0.13 & 2 & 9.1 & $6.87 \times 10^{-3}$ & 0.29 \\
\hline 4 & Alanine, aspartate, and glutamate metabolism & 28 & 0.17 & 2 & 7.1 & $1.10 \times 10^{-2}$ & 0.00 \\
\hline 6 & Glycine, serine, and threonine metabolism & 34 & 0.20 & 2 & 5.9 & $1.61 \times 10^{-2}$ & 0.05 \\
\hline 1 & Synthesis and degradation of ketone bodies & 5 & 0.03 & 1 & 20.0 & $2.95 \times 10^{-2}$ & 0.00 \\
\hline 2 & Taurine and hypotaurine metabolism & 8 & 0.05 & 1 & 12.5 & $4.68 \times 10^{-2}$ & 0.43 \\
\hline 5 & Butanoate metabolism & 15 & 0.09 & 1 & 6.7 & $8.62 \times 10^{-2}$ & 0.00 \\
\hline 7 & Starch and sucrose metabolism & 18 & 0.11 & 1 & 5.6 & $1.03 \times 10^{-1}$ & 0.42 \\
\hline 9 & Selenocompound metabolism & 20 & 0.12 & 1 & 5.0 & $1.13 \times 10^{-1}$ & 0.00 \\
\hline 8 & Citrate cycle (TCA cycle) & 20 & 0.12 & 1 & 5.0 & $1.13 \times 10^{-1}$ & 0.05 \\
\hline 10 & Glycolysis/gluconeogenesis & 26 & 0.16 & 1 & 3.8 & $1.45 \times 10^{-1}$ & 0.10 \\
\hline 11 & Galactose metabolism & 27 & 0.16 & 1 & 3.7 & $1.50 \times 10^{-1}$ & 0.03 \\
\hline 12 & Glyoxylate and dicarboxylate metabolism & 32 & 0.19 & 1 & 3.1 & $1.76 \times 10^{-1}$ & 0.00 \\
\hline 13 & Cysteine and methionine metabolism & 33 & 0.20 & 1 & 3.0 & $1.81 \times 10^{-1}$ & 0.00 \\
\hline 14 & Arginine and proline metabolism & 38 & 0.23 & 1 & 2.6 & $2.01 \times 10^{-1}$ & 0.00 \\
\hline 15 & Tyrosine metabolism & 42 & 0.25 & 1 & 2.4 & $2.24 \times 10^{-1}$ & 0.00 \\
\hline 16 & Primary bile acid biosynthesis & 46 & 0.27 & 1 & 2.2 & $2.44 \times 10^{-1}$ & 0.02 \\
\hline 17 & Aminoacyl-tRNA biosynthesis & 48 & 0.29 & 1 & 2.1 & $2.53 \times 10^{-1}$ & 0.00 \\
\hline
\end{tabular}

* Pathways are ranked by percent compound found in each pathway, which was determined by dividing the number of hits by the total number of compounds in the pathway. ${ }^{*}$ The number of the expected compounds was determined by overrepresentation analysis in MetPA, which uses hypergeometric testing to calculate the number of compounds expected to be in each pathway by chance alone. ${ }^{*}$ Raw $p$ values were determined based on the number of hits and total number of compounds in the pathway. ${ }^{*}$ The pathway impact was found using relative betweenness centrality pathway topology analysis, which calculates metabolite importance based on their position in the pathways.

in treated rats during eight weeks of treatment period $[8,21]$. Karimi et al. [27] reported that normal rats administrated daily with a high dose of aqueous extract saffron stigma $(0.16,0.32$, and $0.48 \mathrm{~g} / \mathrm{kg})$ for two weeks showed normochromic normocytic anaemia. A study done by Bahmani et al. [28] also had investigated saffron toxicity in different doses $(500,1000$, and $2000 \mathrm{mg} / \mathrm{kg} /$ day) in newborn mice, and results showed slight histopathology changes in the mice kidney. Modaghegh et al. [29] also demonstrated that the consumption of $200 \mathrm{mg}$ and $400 \mathrm{mg}$ of saffron tablets in healthy volunteers changed some haematological and biochemical parameters. However, these changes were still in a normal range which does not sound important clinically.

Moreover, Hosseinzadeh et al. [18] revealed that crocin supplementation up to $3 \mathrm{~g} / \mathrm{kg}$ in mice and $15-180 \mathrm{mg} / \mathrm{kg}$ in rats did not cause damage to any major organ in the body. Besides, an elevated liver enzyme profile such as aspartate transaminase (AST) and alanine transaminase (ALT) was noted in both rat groups treated with saffron extract and crocin [30]. However, the level of these enzymes is still lower than the OB group. An increase in the liver enzymes can indicate the toxicity in the body. Nonetheless, it is well known that consumption of foreign material (i.e., herbs) and drugs can increase the liver enzyme level, as the body tried to detoxify the compounds or toxins from the body via the liver.
As mentioned in Materials and Methods, this study revealed that treatment by crocin and saffron extract works both directly and indirectly in changing and altering the biochemistry profile and slowing the rate of weight gain in the obese rats. The treatment managed to suppress the food intake in OBSF80 and OBCR80. Even though there was a favourable trend in weight loss in the treated groups, there was no significant weight loss $(p>0.05)$ found in the treated groups. Thus, the improvement in the biochemistry result indicated that both crocin and saffron extract treatment works in both ways, directly (i.e., the rats consumed the designated dosage) and indirectly (i.e., by suppressing the food intake) in the obese rats. These results are also supported by the metabolic profile of the obese-treated group analyzed using MVDA. From MVDA, the metabolic profile of serum in obese rats treated with the saffron extract $(80 \mathrm{mg} / \mathrm{kg})$ and crocin $(80 \mathrm{mg} / \mathrm{kg})$ deviated from negative control $(\mathrm{OB})$ compared to obese rats that received crocin $(40 \mathrm{mg} / \mathrm{kg})$ and saffron extract $(40 \mathrm{mg} / \mathrm{kg})$. This result indicated that the bioactivities of the saffron extract $(80 \mathrm{mg} / \mathrm{kg})$ and crocin $(80 \mathrm{mg} / \mathrm{kg})$ are dose-dependent, and it could effectively improve the disturbed metabolism in obese rats. To investigate the variation of serum metabolites in obesity and treatment with saffron extract and crocin, a ${ }^{1} \mathrm{H}-\mathrm{NMR}$ metabolomics technique coupled with MVDA was performed to examine and identify potential biomarkers. The potential biomarkers in rat serum identified 
TABLE 3: List of pathways found by qualitative MetPA analysis using MetaboAnalyst 4.0 in OBCR80.

\begin{tabular}{lcccccccc}
\hline Ranking & Pathway & Total & Expected & Hits & \% found & Raw $p$ & Impact \\
\hline 4 & Glycine, serine, and threonine metabolism & 34 & 0.14 & 2 & 5.9 & $6.98 \times 10^{-3}$ & 0.05 \\
1 & Synthesis and degradation of ketone bodies & 5 & 0.02 & 1 & 20.0 & $1.97 \times 10^{-2}$ & 0.00 \\
2 & Taurine and hypotaurine metabolism & 8 & 0.03 & 1 & 12.5 & $3.14 \times 10^{-2}$ & 0.43 \\
3 & Butanoate metabolism & 15 & 0.06 & 1 & 6.7 & $5.83 \times 10^{-2}$ & 0.00 \\
5 & Starch and sucrose metabolism & 18 & 0.07 & 1 & 5.6 & $6.96 \times 10^{-2}$ & 0.42 \\
6 & Citrate cycle (TCA cycle) & 20 & 0.08 & 1 & 5.0 & $7.71 \times 10^{-2}$ & 0.05 \\
7 & Pyruvate metabolism & 22 & 0.09 & 1 & 4.5 & $8.44 \times 10^{-2}$ & 0.21 \\
8 & Glycolysis/gluconeogenesis & 26 & 0.10 & 1 & 3.8 & $9.92 \times 10^{-2}$ & 0.10 \\
9 & Galactose metabolism & 27 & 0.11 & 1 & 3.7 & $1.03 \times 10^{-1}$ & 0.03 \\
10 & Alanine, aspartate, and glutamate metabolism & 28 & 0.11 & 1 & 3.6 & $1.06 \times 10^{-1}$ & 0.00 \\
11 & Glyoxylate and dicarboxylate metabolism & 32 & 0.13 & 1 & 3.1 & $1.21 \times 10^{-1}$ & 0.00 \\
12 & Cysteine and methionine metabolism & 33 & 0.13 & 1 & 3.0 & $1.24 \times 10^{-1}$ & 0.00 \\
13 & Arginine and proline metabolism & 38 & 0.15 & 1 & 2.6 & $1.42 \times 10^{-1}$ & 0.00 \\
14 & Tyrosine metabolism & 42 & 0.17 & 1 & 2.4 & $1.56 \times 10^{-1}$ & 0.00 \\
15 & Primary bile acid biosynthesis & 46 & 0.18 & 1 & 2.2 & $1.70 \times 10^{-1}$ & 0.02 \\
\hline
\end{tabular}

${ }^{*}$ Pathways are ranked by percent compound found in each pathway, which was determined by dividing the number of hits by the total number of compounds in the pathway. ${ }^{*}$ The number of the expected compounds was determined by overrepresentation analysis in MetPA, which uses hypergeometric testing to calculate the number of compounds expected to be in each pathway by chance alone. ${ }^{*}$ Raw $p$ values were determined based on the number of hits and the total number of compounds in the pathway. ${ }^{*}$ The pathway impact was found using relative betweenness centrality pathway topology analysis, which calculates metabolite importance based on their position in the pathways.

by ${ }^{1} \mathrm{H}-\mathrm{NMR}$ and their variation among groups are summarized in Table 1.

\subsection{Effect of Saffron Extract and Crocin on Carbohydrate} Metabolism. Glucose is the primary source of energy for most organisms. It is broken down into pyruvate through the glycolysis metabolic pathway in the cytoplasm (Figure 6). Under aerobic conditions, pyruvate is converted into acetyl coenzyme A (CoA) by the pyruvate dehydrogenase complex (PDC), the entry point into the TCA cycle, whereas in anaerobic conditions, the pyruvate is converted into lactate [31, 32]. Therefore, the disturbance in these metabolite levels indicates glucose homeostasis and pyruvate metabolism imbalance. From this study, a lower level of pyruvate and higher levels of glucose in the OB group, as opposed to the LN group, were in line with other reports on metabolomics researches in HFD-induced obesity models [33-35]. This result is supported by the higher levels of lactate and alanine, which implies that the rate of glycolysis was inhibited and gluconeogenesis was activated [35].

Eight weeks of treatment with either saffron or crocin at $80 \mathrm{mg} / \mathrm{kg}$ managed to significantly $(p<0.05)$ reduce glucose and increase pyruvate level in the treated obese group (Table 1, Figure 6). This is in line with biochemical data in our previous study $[8,21]$ where OBCR 80 and OBSF80 significantly managed to reduce their fasting blood glucose as compared to the OB group. A study was done by Hoshyar et al. [14] which also reported that oral administration of saffron stigma extract in obese rats significantly decreased the level of fasting blood glucose, insulin, and leptin. More- over, a randomized, double-blind, and placebo-controlled study proved that the administration of saffron in eight weeks significantly reduced the concentration of fasting blood glucose and HbAlc in obese-diabetic patient [36]. A decreased level of glucose in obese rats treated with saffron extract and crocin could be related to SREBP-1c and ChREBP activity that is responsible in regulating carbohydrate metabolism (glycolysis and glycogen synthesis) and lipogenesis (triglycerides synthesis) in the liver [37].

Interestingly, only OBSF80 managed to revert the lactate and alanine levels to the same level as LN. These findings were consistent with our previous work [30], where supplementation of saffron extracts $(80 \mathrm{mg} / \mathrm{kg})$ in obese rats significantly reduced the activity of alanine transferase (ALT) close to the lean group which could exert protection against liver tissue damage caused by the consumption of HFD. Previously, it was reported that high accumulation of lactate and alanine in serum indicates impairment of lactate dehydrogenase $(\mathrm{LDH})$ and ALT, where $\mathrm{LDH}$ is an enzyme that is involved in the conversion of acetyl-CoA to lactate and ALT is an enzyme that participates in acetyl-CoA conversion to alanine $[25,26]$. Not to mention, increased HFD consumption can increase the production of acetyl-CoA, which may subsequently exert negative feedback on the activity of PDC [26], thereby inhibiting pyruvate conversion into acetyl CoA and shifting to the high production of lactate and alanine.

Bioactivities exerted by the saffron extract may be due to the presence of safranal (third most abundant bioactive compound) rather than crocin in the saffron extract. The previous study showed that safranal had hypoglycemic 
properties in lowering fasting blood glucose and $\mathrm{HbA} 1 \mathrm{c}$ level and increasing insulin level in diabetic rats [11]. It was indicated that obese rat treated with saffron extract effectively revert metabolites in carbohydrate metabolism such as glucose, lactate, alanine, and pyruvate close to the lean rats suggesting that gluconeogenesis was inhibited, promoting the glucose-alanine and Cori cycle and glycolysis occurs. Moreover, the reduction of alanine caused by saffron extracts suggested that muscle protein and liver gluconeogenesis were inhibited in obese rats. The saffron extract was effective in reverting metabolites in carbohydrate metabolism. Thus, we can conclude that saffron at $80 \mathrm{mg} / \mathrm{kg}$ works better in glucose metabolism compared to crocin.

4.3. Effect of Saffron Extract and Crocin on Lipid Metabolism. Another metabolite, which is strongly associated with obesity, is 3-hydroxybutyrate (3-HB). The present study showed that the $\mathrm{OB}$ group significantly had lower levels of $3-\mathrm{HB}$ in serum as compared to the LN group. This result was also in line with previous studies, where the concentration 3-HB and the rate of $3-\mathrm{HB}$ oxidation in the muscle of the obese group were lower than the lean subjects [33, 38-40]. This is because a decrease in the oxidation of ketone bodies in muscle causes the abnormalities in the muscle metabolism of obese subjects, which is associated with a reduced number and increased mitochondrial dysfunction [38-40].

From the result, both treatments (saffron and crocin) managed to increase 3-HB level compared to the OB group. However, the increment of 3-HB level in OBSF80 was 1.7fold greater than the LN group. Compared to glucose, 3-HB undergoes a different pathway and it has been used as an energy source in an individual that is fasting or has prolonged exercise [41]. Fisler et al. [42] stated that 3-hydroxybutyrate in the blood is related to the reduced diet intake in obese subjects. These findings are similar to our previous study, where there was a significant difference in food intake in saffron and crocin [8]. Previously, saffron was reported to increase satiety in obese individuals, hence causing a reduction in the food intake [8]. Therefore, factors such as fasting and reduced food intake can contribute to the upregulation of 3-HB levels in serum.

4.4. Effect of Saffron Extract and Crocin on Amino Acid Metabolism. Taurine, also known as 2-aminoathane sulfonic acid, is a $\beta$-amino acid that differs from other amino acids, with a sulfonic group rather than a carboxylic group. It is also called a conditional essential amino acid because it can be supplied by dietary ingestion, as well as biochemically synthesized from cysteine catalyzed by cysteine dioxygenase (CDO) in the liver [43]. The present study showed that the OB group had significantly lower levels of taurine compared to the LN group, which is similarly reported in several previous studies $[33,38]$. Taurine was involved in glucose and lipid metabolism. As a result, deficiency of taurine may disturb the activity of glucose and lipid metabolism, thus accelerating the development of obesity [38]. In this study, the lower levels of taurine in obese rats suggest that the inhibition of fatty acid oxidation and reduction of CDO expression are related to obesity as observed by the increased concentration of TC and LDL with a low level of HDL and adiponectin as reported in our previous study $[8,21]$.

An elevated taurine level was noticeable in an obese model after the administration of saffron extract and crocin. Our previous study had reported that saffron extract and crocin have a hypolipidemic effect and hepatoprotective effect against blood lipid and nonalcoholic fatty liver disease $[8,21,30]$. Hoshyar et al. [14] also demonstrated the same observation with our biochemical data where supplementation of saffron extracts (40 and $80 \mathrm{mg} / \mathrm{kg}$ ) in obese rats significantly decreased the serum level of TG, TC, LDL, and antioxidant activity. Besides, a clinical trial study had proved that the intervention of saffron extract $(30 \mathrm{mg})$ for three months significantly decreased the level of TC and LDL in a diabetes patient [44]. This study suggested that the antioxidant properties of saffron extract and crocin may effectively increase the CDO expression of obese rats by promoting taurine synthesis in the liver and adipocytes. The cholesterol-lowering effect of taurine in obese rats treated with saffron extract and crocin could be associated with CYP7A1, the biomarker for cholesterol involved in the regulatory mechanism of cholesterol and bile acid homeostasis and controlled by various factors and nuclear receptors [45]. You et al. [46] also described that body fat loss in obese subjects supplemented with taurine was associated with an increase in serum adiponectin that could promote fatty acid oxidation. This study indicated that synthesis of cholesterol was suppressed after the supplementation of saffron extract and crocin which could be due to the elevation of the taurine level. The increased level of adiponectin also is suggesting that glucose metabolism and fatty acid oxidation took place after administration of saffron extract and crocin in obese rats.

4.5. Effect of Saffron Extract and Crocin on Choline Metabolism. Betaine is an essential osmolyte in cell volume and plays a critical role in methionine recycling pathways by producing a key factor, carnitine, involved in the mechanisms of energy production by transporting long-chain fatty acids into the mitochondria [47]. In the present study, an elevated level of betaine was detected in the OB group. Betaine has been noted to be one of the biomarkers for obesity. It is involved in pathways for methionine recycling in the liver and the regeneration of S-adenosylhomocysteine from homocysteine [33].

Both saffron extract and crocin managed to downregulate the betaine level and prevented the development of steatosis in the liver and lipid accumulation in adipose tissues, where it promotes physiological benefits against cardiovascular diseases. Higher betaine level in an obese animal model can influence the flow of methionine recycling pathways. It causes an elevation of homocysteine levels in the liver, which is considered to be a risk factor of cardiovascular diseases. The higher homocysteine concentration also may relate with hyperlipidemia. The hypomethylation that resulted from the elevated homocysteine level had increased the cholesterol uptake and triglyceride production in the liver, which in the end caused the accumulation of transported fatty acids into adipose tissues [48]. 
4.6. Effect of Saffron Extract and Crocin on Gut Microbiota Metabolism. Trimethylamine N-oxide (TMAO) is a molecule produced from di-, trimethylamine (TMA) and carnitine generated from choline metabolism and gut microbial metabolism [49]. TMAO is also related to functions of the gut microbiota [50]. In this study, higher TMAO level was detected in the OB group. The higher level of TMAO in the blood and urine was attributable to the diet intake containing lecithin and carnitine [51]. These types of foods had stimulated and enabled some microbes in the human gut to convert these metabolites into TMAO $[52,53]$. The administration of saffron extract and crocin in the obese group had significantly decreased the TMAO concentration as compared to that in the OB group. Since dysregulation in TMAO has been associated with impairment in glucose tolerance especially in hepatic insulin signalling pathways [54], this result suggests that the administration of saffron and crocin can prevent the inflammatory response in adipose tissues, which is related to glucose impairment-related diseases.

4.7. Effect of Saffron Extract and Crocin on Creatinine Metabolism. Creatinine is a breakdown product of creatine phosphate in the muscle, which is biosynthesized from arginine and glycine [55]. Obesity is characterized by the elevation of creatinine levels in the blood, as reported in previous studies $[33,56]$ which the present study also reported the same observation. A study done by Matsuzawa-Nagata [50] reported that mice fed an HFD had a higher level of reactive oxygen species (ROS) which causes oxidative stress in the liver and adipose tissues and is a key of triggering insulin resistance and obesity. High creatinine levels also have been described in correlation with oxidative stress [57]. Poor creatinine clearance is also linked to weight gain and central obesity, which causes an increase in metabolic abnormalities as risk factors [52].

Administration of saffron extracts in obese groups significantly decreased the creatinine level compared to that in the negative control group. However, the crocin-treated obese group was unable to have an improved creatinine level as opposed to the normal group. Better creatinine levels were found in the saffron-treated group compared to the crocin-treated group which is due to the presence of safranal. Previous studies had revealed that safranal exerts a protective effect against nephrotoxicity in rats [58, 59]. Saffron-rich antioxidant had lowered creatinine level by decreasing oxidative stress and may effectively improve kidney function in an obese model and prevent any further renal diseases caused by massive obesity.

\section{Conclusions}

Both saffron and crocin at $80 \mathrm{mg} / \mathrm{kg}$ managed to improve metabolite levels such as glucose, pyruvate, betaine, and taurine. Comparison between the two treatments revealed that saffron extract managed to downregulate metabolites such as lactate, alanine, and creatinine. It also managed to upregulate 3-hydroxybutyrate higher than in lean and crocin-treated groups. Illustrated pathway reveals that saffron works mainly by targeting glucose metabolism, whereas crocin works mod- erately on glucose metabolism by regulating glucose and pyruvate level. However, the main factor that differentiates saffron and crocin was their mechanism in lipid metabolism, especially in ketone body production. Even though results from the pattern recognition and previous biochemical analysis revealed that crocin at $80 \mathrm{mg} / \mathrm{kg}$ has better hypolipidemic activities than crocin, this result showed that saffron could also be an excellent alternative to crocin.

\section{Data Availability}

The data used to support the findings of this study are included within article and supplementary information file.

\section{Conflicts of Interest}

The authors declare that there is no conflict of interest regarding the publication of this paper.

\section{Acknowledgments}

The authors would like to thank the management and laboratory staffs of the Faculty of Medicine \& Health Sciences, Universiti Putra Malaysia and Institute of Bioscience, Universiti Putra Malaysia, for their help throughout the study. This research was funded by the Ministry of Science, Technology and Innovation (grant number 06-01-04-SF 1881) and the APC was funded by the Research Management Center, Universiti Putra Malaysia.

\section{Supplementary Materials}

Supplementary Table S1: a composition of the diets fed to the animals. Supplementary Table S2: effect of saffron extract and crocin on the changes of food consumption at initial and the end of 8 weeks. Supplementary Table S3: effect of saffron extract and crocin on biochemical parameters. Supplementary Table S4: ${ }^{1} \mathrm{H}-\mathrm{NMR}$ assignments of metabolites in rat serum. Supplementary Tables S5-S7: the misclassification table for the PLS-DA model of animal groups. Supplementary Figure S1: effect of saffron extract and crocin on body weight during 8 weeks of treatment. Supplementary Figure S2: a typical $500 \mathrm{MHz}{ }^{1} \mathrm{H}-\mathrm{NMR}$ CPMG spectra of serum collected from the lean group (A), the obese group (B), the obese+crocin $80 \mathrm{mg} / \mathrm{kg}$ group (C), and the obese +saffron extract $80 \mathrm{mg} / \mathrm{kg}$ group (D). Supplementary Figures S3-S14: the permutation test (100 cycles) for the PLS-DA model of animal groups. (Supplementary Materials)

\section{References}

[1] WHO, Obesity, Fact Sheet, World Health Organization, Geneva, Switzerland, 2016.

[2] D. Rucker, R. Padwal, S. K. Li, C. Curioni, and D. C. Lau, "Long term pharmacotherapy for obesity and overweight: updated meta-analysis," BMJ, vol. 335, no. 7631, pp. 1194-1199, 2007.

[3] J. W. Yun, "Possible anti-obesity therapeutics from nature - A review," Phytochemistry, vol. 71, no. 14-15, pp. 1625-1641, 2010 . 
[4] I. Vermaak, A. M. Viljoen, and J. H. Hamman, "Natural products in anti-obesity therapy," Natural Product Reports, vol. 28, no. 9, pp. 1493-1533, 2011.

[5] S. Z. Mousavi and S. Z. Bathaie, "Historical uses of saffron: identifying potential new avenues for modern research," Avicenna Journal of Phytomedicine, vol. 1, no. 2, pp. 5766, 2011.

[6] J. L. Rios, M. C. Recio, R. M. Giner, and S. Manez, "An update review of saffron and its active constituents," Phytotherapy Research, vol. 10, no. 3, pp. 189-193, 1996.

[7] M. Mashmoul, A. Azlan, H. Khaza'ai, B. Yusof, and S. Noor, "Saffron: a natural potent antioxidant as a promising antiobesity drug," Antioxidants, vol. 2, no. 4, pp. 293-308, 2013.

[8] M. Mashmoul, A. Azlan, B. N. M. Yusof, H. Khaza'ai, N. Mohtarrudin, and M. T. Boroushaki, "Effects of saffron extract and crocin on anthropometrical, nutritional and lipid profile parameters of rats fed a high fat diet," Journal of Functional Foods, vol. 8, pp. 180-187, 2014.

[9] A. Amin, A. A. Hamza, K. Bajbouj, S. S. Ashraf, and S. Daoud, "Saffron: a potential candidate for a novel anticancer drug against hepatocellular carcinoma," Hepatology, vol. 54, no. 3, pp. 857-867, 2011.

[10] A. N. Assimopoulou, Z. Sinakos, and V. P. Papageorgiou, "Radical scavenging activity of Crocus sativus L. extract and its bioactive constituents," Phytotherapy Research, vol. 19, no. 11, pp. 997-1000, 2005.

[11] S. Kianbakht and R. Hajiaghaee, "Anti-hyperglycemic effects of saffron and its active constituents, crocin and safranal, in alloxan-induced diabetic rats," Journal of Medicinal Plant, vol. 3, no. 39, pp. 82-89, 2011.

[12] E. Moshiri, A. A. Basti, A. A. Noorbala, A. H. Jamshidi, S. Hesameddin Abbasi, and S. Akhondzadeh, "Crocus sativus L. (petal) in the treatment of mild-to-moderate depression: A double-blind, randomized and placebo-controlled trial," Phytomedicine, vol. 13, no. 9-10, pp. 607-611, 2006.

[13] M. Hemmati, E. Zohoori, O. Mehrpour et al., "Anti-atherogenic potential of jujube, saffron and barberry: anti-diabetic and antioxidant actions," EXCLI Journal, vol. 14, pp. 908915, 2015.

[14] R. Hoshyar, M. Hosseinian, M. Rajabian Naghandar et al., "Anti-dyslipidemic properties of saffron: reduction in the associated risks of atherosclerosis and insulin resistance," Iranian Red Crescent Medical Journal, vol. 18, no. 12, 2016.

[15] M. A. Papandreou, M. Tsachaki, S. Efthimiopoulos, P. Cordopatis, F. N. Lamari, and M. Margarity, "Memory enhancing effects of saffron in aged mice are correlated with antioxidant protection," Behavioural Brain Research, vol. 219, no. 2, pp. 197-204, 2011.

[16] J. K. Nicholson and J. C. Lindon, "Systems biology: metabonomics," Nature, vol. 455, no. 7216, pp. 1054-1056, 2008.

[17] Y. Dai, Z. Li, L. Xue et al., "Metabolomics study on the antidepression effect of xiaoyaosan on rat model of chronic unpredictable mild stress," Journal of Ethnopharmacology, vol. 128, no. 2, pp. 482-489, 2010.

[18] H. Hosseinzadeh, V. M. Shariaty, A. K. Sameni, and M. Vahabzadeh, "Acute and sub-acute toxicity of crocin, a constituent of Crocus sativus L. (saffron), in mice and rats," Pharmacology, vol. 2, pp. 943-951, 2010.

[19] S. M. Moosavi, M. Ahmadi, M. Amini, and B. Vazirzadeh, "The effects of 40 and $80 \mathrm{mg}$ hydro-alcoholic extract of Crocus sativus in the treatment of mild to moderate depression," Jour- nal of Mazandaran University of Medical Sciences, vol. 24, no. 113, pp. 48-53, 2014.

[20] O. Beckonert, H. C. Keun, T. M. Ebbels et al., "Metabolic profiling, metabolomic and metabonomic procedures for NMR spectroscopy of urine, plasma, serum and tissue extracts," Nature Protocols, vol. 2, no. 11, pp. 2692-2703, 2007.

[21] M. Mashmoul, A. Azlan, N. Mohtarrudin, B. N. M. Yusof, and H. Khaza'ai, "Saffron extract and crocin reduced biomarkers associated with obesity in rats fed a high-fat diet," Malaysian Journal of Nutrition, vol. 23, no. 1, pp. 117-127, 2017.

[22] M. DiGirolamo, F. D. Newby, and J. Lovejoy, "Lactate production in adipose tissue: a regulated function with extra-adipose implications," The FASEB Journal, vol. 6, no. 7, pp. 2405-2412, 1992.

[23] M. Kanehisa, Y. Sato, M. Kawashima, M. Furumichi, and M. Tanabe, "KEGG as a reference resource for gene and protein annotation," Nucleic Acids Research, vol. 44, no. D1, pp. D457-D462, 2015.

[24] J. Chong, O. Soufan, C. Li et al., "MetaboAnalyst 4.0: towards more transparent and integrative metabolomics analysis," Nucleic Acids Research, vol. 46, no. W1, pp. W486-W494, 2018.

[25] J. Xia, I. V. Sinelnikov, B. Han, and D. S. Wishart, "MetaboAnalyst 3.0-making metabolomics more meaningful," Nucleic Acids Research, vol. 43, no. W1, pp. W251-W257, 2015.

[26] N. Abedimanesh, S. Z. Bathaie, S. Abedimanesh, B. Motlagh, A. Separham, and A. Ostadrahimi, "Saffron and crocin improved appetite, dietary intakes and body composition in patients with coronary artery disease," Journal of Cardiovascular and Thoracic Research, vol. 9, no. 4, pp. 200-208, 2017.

[27] G. H. N. Karimi, H. Taiebi, H. Hosseinzadeh, and F. Shirzad, "Evaluation of subacute toxicity of aqueous extract of Crocus sativus L. stigma and petal in rats," Journal of Medicinal Plants, vol. 4, no. 12, pp. 29-35, 2004.

[28] M. Bahmani, M. Rafieian, A. Baradaran, S. Rafieian, and M. Rafieian-kopaei, "Nephrotoxicity and hepatotoxicity evaluation of Crocus sativus stigmas in neonates of nursing mice," Journal of Nephropathology, vol. 3, no. 2, pp. 81-85, 2014.

[29] M. H. Modaghegh, M. Shahabian, H. A. Esmaeili, O. Rajbai, and H. Hosseinzadeh, "Safety evaluation of saffron (Crocus sativus) tablets in healthy volunteers," Phytomedicine, vol. 15, no. 12, pp. 1032-1037, 2008.

[30] M. Mashmoul, A. Azlan, N. Mohtarrudin et al., "Protective effects of saffron extract and crocin supplementation on fatty liver tissue of high-fat diet-induced obese rats," BMC Complementary and Alternative Medicine, vol. 16, no. 1, pp. 401-408, 2016.

[31] M. H. Ryu and Y. S. Cha, "The effects of a high-fat or highsucrose diet on serum lipid profiles, hepatic acyl-CoA synthetase, carnitine palmitoyltransferase-I, and the acetyl-CoA carboxylase mRNA levels in rats," BMB Reports, vol. 36, no. 3, pp. 312-318, 2003.

[32] M. Mayr, Y. L. Chung, U. Mayr et al., "Proteomic and metabolomic analyses of atherosclerotic vessels from apolipoprotein E-deficient mice reveal alterations in inflammation, oxidative stress, and energy metabolism," Arteriosclerosis, Thrombosis, and Vascular Biology, vol. 25, no. 10, pp. 2135-2142, 2005.

[33] N. G. S. Jambocus, N. Saari, A. Ismail, A. Khatib, M. F. Mahomoodally, and A. A. Hamid, "An investigation into the antiobesity effects of Morinda citrifolia L. leaf extract in high fat diet induced obese rats using a ${ }^{1} \mathrm{H}-\mathrm{NMR}$ 
metabolomics approach," Journal of Diabetes Research, vol. 2016, Article ID 2391592, 14 pages, 2016.

[34] N. J. Serkova, M. Jackman, J. L. Brown et al., "Metabolic profiling of livers and blood from obese Zucker rats," Journal of Hepatology, vol. 44, no. 5, pp. 956-962, 2006.

[35] X. Song, J. Wang, P. Wang, N. Tian, M. Yang, and L. Kong,

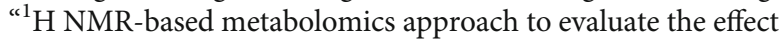
of Xue-Fu-Zhu-Yu decoction on hyperlipidemia rats induced by high-fat diet," Journal of Pharmaceutical and Biomedical Analysis, vol. 78-79, pp. 202-210, 2013.

[36] E. Karimi-Nazari, A. Nadjarzadeh, R. Masoumi et al., "Effect of saffron (Crocus sativus L.) on lipid profile, glycemic indices and antioxidant status among overweight/obese prediabetic individuals: a double-blinded, randomized controlled trial," Clinical nutrition ESPEN, vol. 34, pp. 130-136, 2019.

[37] P. Konstantopoulos, I. P. Doulamis, A. Tzani et al., "Metabolic effects of Crocus sativus and protective action against nonalcoholic fatty liver disease in diabetic rats," Biomedical reports, vol. 6, no. 5, pp. 513-518, 2017.

[38] G. E. Duggan, D. S. Hittel, C. C. Hughey, A. Weljie, H. J. Vogel, and J. Shearer, "Differentiating short- and long-term effects of diet in the obese mouse using ${ }^{1} \mathrm{H}$-nuclear magnetic resonance metabolomics," Diabetes, Obesity and Metabolism, vol. 13, no. 9, pp. 859-862, 2011.

[39] M. S. Klein, C. Dorn, M. Saugspier, C. Hellerbrand, P. J. Oefner, and W. Gronwald, "Discrimination of steatosis and NASH in mice using nuclear magnetic resonance spectroscopy," Metabolomics, vol. 7, no. 2, pp. 237-246, 2011.

[40] I. Rubio-Aliaga, B. d. Roos, M. Sailer et al., "Alterations in hepatic one-carbon metabolism and related pathways following a high-fat dietary intervention," Physiological Genomics, vol. 43, no. 8, pp. 408-416, 2011.

[41] J. C. Newman and E. Verdin, “ $\beta$-Hydroxybutyrate: much more than a metabolite," Diabetes Research and Clinical Practice, vol. 106, no. 2, pp. 173-181, 2014.

[42] J. S. Fisler, M. Egawa, and G. A. Bray, "Peripheral 3hydroxybutyrate and food intake in a model of dietary-fat induced obesity: effect of vagotomy," Physiology \& Behavior, vol. 58, no. 1, pp. 1-7, 1995.

[43] M. Walczewska, M. Ciszek-Lenda, M. Surmiak, A. Kozlowska, S. Jozefowski, and J. Marcinkiewicz, "Impact of taurine on innate and adaptive immunity as the result of $\mathrm{HOCl}$ neutralization," in In Taurine 9, pp. 109-120, Springer International Publishing, 2015.

[44] A. Moravej Aleali, R. Amani, H. Shahbazian, F. Namjooyan, S. M. Latifi, and B. Cheraghian, "The effect of hydroalcoholic saffron (Crocus sativus L.) extract on fasting plasma glucose, HbAlc, lipid profile, liver, and renal function tests in patients with type 2 diabetes mellitus: a randomized double-blind clinical trial," Phytotherapy Research, vol. 33, no. 6, pp. 1648-1657, 2019.

[45] W. Chen, J. X. Guo, and P. Chang, "The effect of taurine on cholesterol metabolism," Molecular Nutrition \& Food Research, vol. 56, no. 5, pp. 681-690, 2012.

[46] J. S. You, J. Y. Park, X. Zhao, J. S. Jeong, M. J. Choi, and K. J. Chang, "Relationship among serum taurine, serum adipokines, and body composition during 8-week human body weight control program," in In Taurine 8, pp. 113-120, Springer, New York, NY, USA, 2013.

[47] M. Lever, P. M. George, W. Atkinson et al., "Plasma lipids and betaine are related in an acute coronary syndrome cohort," PLoS One, vol. 6, no. 7, article e21666, 2011.
[48] M. Wang, R.-J. A. N. Lamers, H. A. A. J. Korthout et al., "Metabolomics in the context of systems biology: bridging traditional Chinese medicine and molecular pharmacology," Phytotherapy Research, vol. 19, no. 3, pp. 173-182, 2005.

[49] M. Al-Waiz, S. Mikov, C. Mitchell, and R. L. Smith, "The exogenous origin of trimethylamine in the mouse," Metabolism, vol. 41, no. 2, pp. 135-136, 1992.

[50] N. Matsuzawa-Nagata, T. Takamura, H. Ando et al., "Increased oxidative stress precedes the onset of high-fat diet-induced insulin resistance and obesity," Metabolism, vol. 57, no. 8, pp. 1071-1077, 2008.

[51] C. Y. Jiang, K. M. Yang, L. Yang, Z. X. Miao, Y. H. Wang, and H. B. Zhu, "A ${ }^{1} \mathrm{H}$ NMR-based metabonomic investigation of time-related metabolic trajectories of the plasma, urine and liver extracts of hyperlipidemic hamsters," PLoS One, vol. 8, no. 6, p. e66786, 2013.

[52] H. J. Kim, J. H. Kim, S. Noh et al., "Metabolomic analysis of livers and serum from high-fat diet induced obese mice," Journal of Proteome Research, vol. 10, no. 2, pp. 722-731, 2010.

[53] R. A. Koeth, Z. Wang, B. S. Levison et al., "Intestinal microbiota metabolism of L-carnitine, a nutrient in red meat, promotes atherosclerosis," Nature Medicine, vol. 19, no. 5, pp. 576-585, 2013.

[54] X. Gao, X. Liu, J. Xu, C. Xue, Y. Xue, and Y. Wang, "Dietary trimethylamine $\mathrm{N}$-oxide exacerbates impaired glucose tolerance in mice fed a high fat diet," Journal of Bioscience and Bioengineering, vol. 118, no. 4, pp. 476-481, 2014.

[55] J. Hjelmesæth, J. Røislien, N. Nordstrand, D. Hofs $\varnothing$, H. Hager, and A. Hartmann, "Low serum creatinine is associated with type 2 diabetes in morbidly obese women and men: a crosssectional study," BMC Endocrine Disorders, vol. 10, 2010.

[56] J.-Y. Jung, I.-Y. Kim, Y.-N. Kim et al., "1 H NMR-based metabolite profiling of diet-induced obesity in a mouse mode," BMB Reports, vol. 45, no. 7, pp. 419-424, 2012.

[57] Y. Wang, H. Tang, J. K. Nicholson, P. J. Hylands, J. Sampson, and E. Holmes, "A metabonomic strategy for the detection of the metabolic effects of chamomile (Matricaria recutita L.) ingestion," Journal of Agricultural and Food Chemistry, vol. 53, no. 2, pp. 191-196, 2005.

[58] M. T. Boroushaki and H. R. Sadeghnia, "Protective effect of safranal against gentamicin-induced nephrotoxicity in rat," Iranian Journal of Medical Sciences, vol. 34, no. 4, pp. 285288, 2015.

[59] A. T. Hariri, S. A. Moallem, M. Mahmoudi, B. Memar, and H. Hosseinzadeh, "Sub-acute effects of diazinon on biochemical indices and specific biomarkers in rats: protective effects of crocin and safranal," Food and Chemical Toxicology, vol. 48, no. 10 , pp. 2803-2808, 2010. 